\title{
Feline immunodeficiency virus (FIV) env recombinants are common in natural infections
}

\author{
Paweł M Bęczkowski ${ }^{1,2^{*}}$, Joseph Hughes ${ }^{1}$, Roman Biek ${ }^{1,3}$, Annette Litster ${ }^{4}$, Brian J Willett ${ }^{1}$ and Margaret J Hosie
}

\begin{abstract}
Background: Recombination is a common feature of retroviral biology and one of the most important factors responsible for generating viral diversity at both the intra-host and the population levels. However, relatively little is known about rates and molecular processes of recombination for retroviruses other than HIV, including important model viruses such as feline immunodeficiency virus (FIV).

Results: We investigated recombination in complete FIV env gene sequences $(n=355)$ isolated from 43 naturally infected cats. We demonstrated that recombination is abundant in natural FIV infection, with over $41 \%$ of the cats being infected with viruses containing recombinant env genes. In addition, we identified shared recombination breakpoints; the most significant hotspot occurred between the leader/signal fragment and the remainder of env.

Conclusions: Our results have identified the leader/signal fragment of env as an important site for recombination and highlight potential limitations of the current phylogenetic classification of FIV based on partial env sequences. Furthermore, the presence of abundant recombinant FIV in the USA poses a significant challenge for commercial diagnostic tests and should inform the development of the next generation of FIV vaccines.
\end{abstract}

Keywords: FIV, Feline immunodeficiency virus, Recombination, Leader, Quasispecies, Phylogenetic classification, Natural infection

\section{Background}

Recombination, together with point mutations introduced by the error prone reverse transcriptase (RT) [1] and the activity of host restriction factors [2], is regarded as the most important mechanism for generating genetic diversity among retroviruses [3,4]. Two features of the retroviral life cycle facilitate recombination: 1) the presence of two RNA genomes within each viral particle and 2) the tendency of RT to switch between those RNA molecules during provirus synthesis $[5,6]$. This can result in the synthesis of recombinant DNA of mixed ancestry, originating from both RNA molecules [7]. In HIV-1 infection, recombination occurs between homologous viral variants of similar fitness at an exceptionally high rate [8,9]. However, its significance in generating novel variants increases dramatically when a cell becomes infected with two or more genetically distinct virions [10]. Recombination can occur between virions of the same or different subtypes, resulting in the generation

\footnotetext{
* Correspondence: pawel.beczkowski@glasgow.ac.uk

${ }^{1}$ Centre for Virus Research, University of Glasgow, Glasgow, United Kingdom

${ }^{2}$ Small Animal Hospital, University of Glasgow, Glasgow, United Kingdom

Full list of author information is available at the end of the article
}

of intra- or inter-subtype recombinants, respectively [10]. Recombination can have profound implications on the fitness of the generated viral quasispecies and subsequently on the pathogenesis and clinical outcome of infection [11]. Intra- and inter-subtype recombinants have been identified in HIV infection and unique recombinant forms (URF) with limited transmission can be distinguished from commonly circulating recombinant forms (CRF), where the term CRF applies to recombinant viruses sharing identical mosaic structures that are detected in multiple distinct epidemiological areas [12]. CRFs play an important role in the HIV pandemic, accounting for over $20 \%$ of infections in some countries [13]. In contrast to HIV, much less is known about natural recombination in other retroviruses, including important models such as feline immunodeficiency virus (FIV). Although FIV recombinants have been identified [14-17], the prevalence of recombinant env sequences remains poorly quantified. This applies particularly to the open reading frame of the env gene which, in contrast to primate retroviruses, contains an unusually long leader/signal region [18].

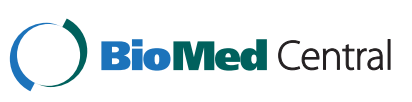

(c) 2014 Bęczkowski et al.; licensee BioMed Central Ltd. This is an Open Access article distributed under the terms of the Creative Commons Attribution License (http://creativecommons.org/licenses/by/4.0), which permits unrestricted use, distribution, and reproduction in any medium, provided the original work is properly credited. The Creative Commons Public Domain Dedication waiver (http://creativecommons.org/publicdomain/zero/1.0/) applies to the data made available in this article, unless otherwise stated. 
FIV is currently assigned to five distinct subtypes, denoted A, B, C, D and E, based on the diversity of the V3-V5 region of the env gene [19]. Subtype A is prevalent in the west coast of the USA and Australia and is the only subtype found in the UK, while subtype B is most prevalent on the east coast of the USA and central and southern Europe [20-24]. Subtype C has been identified in California [20], Canada [14,16] and Taiwan [25] and subtype D in Japan $[19,26]$, while the putative subtype E was reported in Argentina [27]. Hence, despite extensive movement of humans and their cats and subtypes A, B and C being found on multiple continents [14], geographical clustering of FIV (based on the current classification) is still evident. However, the vast majority of published env sequences represent a V3-V5 region of approximately $700 \mathrm{bp}$, which is too short to enable the reliable detection of recombination.

Given that most previous studies focused upon relatively small gene fragments, the occurrence and role of recombination in natural FIV infection could well have been underestimated. The aim of this study was to address this issue by studying the molecular evolution and recombination of FIV in two cohorts of cats in the USA (Memphis, $\mathrm{n}=27$ and Chicago, $\mathrm{n}=16$ ) naturally infected with FIV. By examining FIV env sequences from 43 domestic cats naturally infected with FIV, we aimed to determine a) the prevalence of recombinant env sequences; b) the subtype composition amongst field isolates; and c) the site(s) of common recombination break-points.

\section{Results}

\section{Phylogenetic inference}

A Maximum Likelihood (ML) tree was constructed using the entire data set [see Additional file 1] and examined carefully for evidence of non-monophyletic clustering of multiple sequences isolated from each cat. Intra-host sequences isolated from the majority of cats ( $n=40,93 \%$ ) clustered together, forming monophyletic groups. After excluding data from the three cats with inconsistent phylogenetic assignment, analysis of the tree revealed that sequences from 24 cats clustered together with previously published clade B reference sequences, while sequences from 16 cats were assigned to clade A. The number of env genes classified as clade A (7/14, $50 \%)$ and $\mathrm{B}(7 / 14,50 \%)$ were equally distributed in the Chicago cats, while in the Memphis cohort, a larger proportion of env genes belonged to clade B (17/26, 65\%). Sequences (comprising $7 \%$ of the total) from three cats (M5, P8 and P21) isolated from different time points showed inconsistent phylogenetic assignments, suggesting that they might have been infected with additional viruses, perhaps transmitted from cats not sampled for this study, or that sample sizes at later time points were insufficient to detect the earlier virus.
The ML tree from the entire dataset was used to select a reduced data set for recombination analysis comprising: a) one randomly selected sequence from each cat where the intra-host sequences formed a monophyletic cluster, and b) all sequences from non-monophyletic groups. These sequences were combined with 19 reference sequences and used to construct a further ML tree which was rooted on the reference clade C env sequence [Figure 1].

\section{Recombination}

GARD analysis of the data set $(n=68)$ identified 22 recombinants providing evidence for four breakpoints with significant topological incongruence $(p=0.01)$. A subsequent GARD analysis was run on the data set comprising only reference sequences and previously identified recombinant sequences $(n=22)$. This alignment was spliced by GARD at six breakpoints into seven spans: 1) 1-354; 2) 355-564; 3) 565-1272; 4) 1273-1608; 5) 16091868; 6) 1869-2255 and 7) 2256-2604 [Figure 1]. For each span, a separate ML tree was created. Examination of GARD-determined trees [see Additional file 2: Figure S1] revealed that sequences from 18/26 (69\%) of the Memphis cats showed consistent clade assignment and represented non-recombinant clade B envs, whereas recombinant sequences were identified in the remaining $8 / 26$ (31\%) cats. In the Chicago group, sequences from $7 / 14$ cats $(50 \%)$ were non-recombinant and consistently clustered with the reference clade B env sequences, while the remaining $7 / 14$ sequences $(50 \%)$ were identified as A/B recombinants.

The jpHMM analysis generally supported all of the previous recombination breakpoints identified in GARD, but also suggested the existence of seven additional breakpoints [see Additional file 3: Table S1]. In order to focus on the most strongly supported recombination events, we examined in detail the breakpoints identified by the two methods.

Recombinant sequences had different mosaic compositions and were classified in three groups: 1) sequences from cats M2, M33, M47, M50, P14, P15 and P18 in which the first two spans $(1-354,355-564)$ belonged to clade $\mathrm{B}$, while the remaining five spans consistently clustered as clade A; 2) sequences from cats M8, P4, P5, P7 and P13 where only the second span (355-564) was assigned to clade $\mathrm{B}$ while the remaining spans clustered with clade A; and 3) sequences from cats M20 and M48 in which only the last span (2256-2604) was assigned to clade B, while all other fragments were assigned to clade A. The remaining two recombinant sequences (from cats M31 and M41) did not have a recombination pattern in common with any of the other sequences in the data set [Table 1]. The locations of the recombination hotspots between the leader and the stem of the V1/V2 region 
(See figure on previous page.)

Figure 1 Maximum likelihood (ML) tree based on the 708 bp fragment (span 3 as identified by GARD) of the env sequences and their recombination history as inferred by GARD. The data include 47 entire env nucleotide sequences (representative of a total of 355 sequences from Chicago and Memphis), 15 full length env sequences derived from GenBank: Aomori 1 [D37816], Aomori 2 [D37817.1], FIV C [AF474246.1], Dixon [L00608.1], Dutch [X60725], Fukuoka [D37815.1], Sendai 1 [D37813.1], Shizuoka [D37811.1], UK2 [X69494.1], UK8 [X69496.1], USIL2489

[U11820.1], Yokohama [D37812.1], Petaluma [M25381.1], PPR [M36968.1], Leviano [FJ374696.1], three V3-V5 region sequences representing Clade E: LP3 [D84496], LP20 [D84498], LP24 [D84500] and one shorter 504 bp in length RUS14 [EF447297] sequence. Taxa with inconsistent clade assignment are represented with an asterisk (P8, P21). Non-monophyletic taxa from cat M5 are marked with a triangle. The tree is based on an HKY model, rooted on FIV C sequence and is drawn to scale, with branch lengths measured in substitutions per site. Only bootstrap values above 80 are shown. * represents bootstrap values of 100 .

(approximately position 562-576) were common among recombinants from both cohorts [Table 1]. A similarity plot representative of the Memphis and Chicago recombinants in relation to reference A and $\mathrm{B} e n v$ sequences is shown in [see Additional file 4: Figure S2].

RDP software was used to examine putative parents contributing to the mosaic structure of recombinant sequences. RDP consistently identified the clade A, FIV Dixon strain [GenBank:L00608.1] as a putative major parent and determined Chicago strains P10 and P2 as putative minor parents for M2, M31, M33, M47, M50, P4, P5, P7, P13, P14, P15, P18 and M20, M48, respectively [Table 1].

Recombination analysis of env sequences from three cats carrying non-monophyletic groups of viruses revealed that sequences from cat P8 at time point $\mathrm{C}$ were non-recombinant clade $B$, while time point $A$ sequences were classified as $\mathrm{A} / \mathrm{B}$ recombinants. It is intriguing that all time point $\mathrm{C}$ sequences in this cat contained premature stop codons [see Additional file 5: Table S2]. Taken together with the FIV load data (time point A, 395 genomes/mL; time point $\mathrm{C}, 1061881$ genomes $/ \mathrm{mL}$ [see Additional file 6: Table S3]), it is possible that cat P8 became infected with freshly acquired clade B env virus that outcompeted the recombinant strain. In cat P21, sequences at time point $\mathrm{B}$ appeared to be entirely clade B, while those at time point $C$ showed an incongruent assignment and were identified as clade A/B mosaics [Table 1]. A similar scenario to P8 is possible; in this case, recombinant virus (time point C FIV load, 1077 genomes $/ \mathrm{mL}$ ) could have outcompeted the non-recombinant strain from time point B (FIV load, 45 genomes $/ \mathrm{mL}$ ) [see Additional file 6: Table S3]. In contrast, all sequences from cat M5 were non-recombinant [Figure 1]. Examination of their phylogenetic assignment, considering the shared housing conditions, suggests that one-way transmission might have occurred during the study. Similar to P8 and P21, the original strain could have been outcompeted by newly acquired virus by time point $\mathrm{C}$ (sequential FIV load data were not available for this cat).

There was a notable difference in terms of the abundance of clade A recombinants compared to very few recombinant sequences clustering with clade B sequences [Figure 1]. Based on the ML phylogeny and the recombination analyses of sequences from both cohorts, we propose that the following groups of mosaic viruses represent putative CRFs of FIV: 1) M47, M50 (bootstrap support 96\%), 2) M20, M48 (bootstrap support 100\%), 3) P14, P8A (bootstrap support 100\%). Given that cats in each of those three groups could have previously been involved in territorial fights, it is likely that the proposed CRFs of FIV were transmitted in the field. Although additional clusters of recombinant forms exist, the bootstrap supports were too low to define them confidently.

\section{Discussion}

The phylogenetic relationship of the sequences from Chicago and Memphis cohorts was in agreement with previously published data demonstrating the presence of clade A and clade B viruses in the USA [20]. However, most previous analyses did not account for potential recombination. Our analyses show that env genes with shared recombination breakpoints and with inconsistent clade assignment circulated widely in the Memphis and Chicago cohorts, with over $41 \%$ of tested cats being infected with a recombinant form.

Recombination is an important event in retroviral evolution, which, in cases of super-infection, can lead to the emergence of novel viral variants. Although there was no evidence of more severe clinical manifestations in cats infected with recombinant strains [28], newly created recombinants could potentially exhibit novel pathogenicity compared to the parental strains, for example manifesting more severe clinical outcomes or being transmitted more easily within and among hosts [4]. Cats with outdoor access, especially in areas where the prevalence of FIV is relatively high, are likely to acquire viruses during multiple transmission events. This phenomenon has been documented in experimental FIV infection [29], with one study describing recombination following super-infection [30]. Recombinant sequences in the present study were consistently identified in subsequent follow up samples, suggesting that such genotypes did not arise as a result of either PCR errors or contamination and that the recombinant viruses incurred no fitness costs preventing them from replicating and persisting over several months. Indeed, the high frequency of clade A/B recombinants in our study might indicate that these represent widely 
Table 1 Recombination testing of sequences from Memphis and Chicago

\begin{tabular}{|c|c|c|c|c|c|c|c|c|c|c|}
\hline \multirow{3}{*}{ env } & \multicolumn{7}{|c|}{ GARD determined breakpoints } & \multicolumn{3}{|c|}{ RDP determined recombinants } \\
\hline & S1 & S2 & S3 & S4 & S5 & S6 & S7 & CLADE & MINOR & MAJOR \\
\hline & $1-354$ & $355-564$ & $565-1272$ & $1273-1608$ & $1609-1868$ & $1869-2255$ & 2256-2604 & & & \\
\hline M1 & B & B & B & B & B & B & B & B & NR & NR \\
\hline M2 & B & B & A & A & A & A & A & $A / B$ & P10 & DIXON \\
\hline M3 & B & B & B & B & B & B & B & B & NR & NR \\
\hline M5At & B & B & B & B & B & B & B & B & NR & NR \\
\hline $\mathrm{M} 5 \mathrm{C}+$ & B & B & B & B & B & B & B & B & NR & NR \\
\hline M8 & A & B & A & A & A & A & A & $A / B$ & P10 & DIXON \\
\hline M10 & B & B & B & B & B & B & B & B & NR & NR \\
\hline M11 & B & B & B & B & B & B & B & B & NR & NR \\
\hline M12 & B & B & B & B & B & B & B & B & NR & NR \\
\hline M14 & B & B & B & B & B & B & B & B & NR & NR \\
\hline M15 & B & B & B & B & B & B & B & B & NR & NR \\
\hline M16 & B & B & B & B & B & B & B & B & NR & NR \\
\hline M20 & A & A & A & A & A & A & B & $A / B$ & P2 & DIXON \\
\hline M25 & B & B & B & B & B & B & B & B & NR & NR \\
\hline M26 & B & B & B & B & B & B & B & B & NR & NR \\
\hline M28 & B & B & B & B & B & B & B & B & NR & NR \\
\hline M29 & B & B & B & B & B & B & B & B & NR & NR \\
\hline M30 & B & B & B & B & B & B & B & B & NR & NR \\
\hline M31 & B & B & A & A & A & A & B & $A / B$ & P10 & DIXON \\
\hline M32 & B & B & B & B & B & B & B & B & NR & NR \\
\hline M33 & B & B & A & A & A & A & A & $A / B$ & P10 & DIXON \\
\hline M41 & B & B & A & A & A & D & B & $A / B / D$ & P10 & DIXON \\
\hline M44 & B & B & B & B & B & B & B & B & NR & NR \\
\hline M46 & B & B & B & B & B & B & B & B & NR & NR \\
\hline M47 & B & B & A & A & A & A & A & $A / B$ & P10 & DIXON \\
\hline M48 & A & A & A & A & A & A & B & $A / B$ & P2 & DIXON \\
\hline M49 & B & B & B & B & B & B & B & B & NR & NR \\
\hline M50 & B & B & A & A & A & A & A & $A / B$ & P10 & DIXON \\
\hline P2 & B & B & B & B & B & B & B & B & NR & NR \\
\hline P4 & A & B & A & A & A & A & A & $A / B$ & P10 & DIXON \\
\hline P5 & A & B & A & A & A & A & A & $A / B$ & P10 & DIXON \\
\hline P6 & B & B & B & B & B & B & B & B & NR & NR \\
\hline P7 & A & B & A & A & A & A & A & $A / B$ & P10 & DIXON \\
\hline P8At & B & B & A & A & A & A & A & $A / B$ & P10 & DIXON \\
\hline P8C† & B & B & B & B & B & B & B & B & NR & NR \\
\hline P9 & B & B & B & B & B & B & B & B & NR & NR \\
\hline P10 & B & B & B & B & B & B & B & B & NR & NR \\
\hline P11 & B & B & B & B & B & B & B & B & NR & NR \\
\hline P13 & A & B & A & A & A & A & A & $A / B$ & P10 & DIXON \\
\hline P14 & B & B & A & A & A & A & A & $A / B$ & P10 & DIXON \\
\hline P15 & B & B & A & A & A & A & A & $A / B$ & P10 & DIXON \\
\hline P17 & B & B & B & B & B & $\mathrm{B}$ & $B$ & B & NR & NR \\
\hline
\end{tabular}


Table 1 Recombination testing of sequences from Memphis and Chicago (Continued)

\begin{tabular}{|c|c|c|c|c|c|c|c|c|c|c|}
\hline P18 & B & B & A & A & A & A & $A$ & $\mathrm{~A} / \mathrm{B}$ & P10 & DIXON \\
\hline P21B十 & B & B & B & B & B & B & B & B & $N R$ & NR \\
\hline P21C† & B & B & A & A & B & A & $A$ & $\mathrm{~A} / \mathrm{B}$ & P10 & DIXON \\
\hline P22 & B & B & B & B & B & B & B & B & NR & NR \\
\hline
\end{tabular}

Spans (S1-S7) are assigned as clade A, B and D as inferred by GARD analysis. The final clade assignment following recombination testing is shown in the column "CLADE". Non recombinant sequences are denoted NR. RDP determined major and minor parents for mosaic recombinant sequences are shown in the final two columns. † sequences from the same cat but different time points with previously identified non-monophyletic clustering.

distributed viruses with a significant fitness advantage. A similar pattern of recombination, with a shared break point within gag, was reported in a Canadian study, suggesting the existence of enzootic recombinant forms circulating in Ontario [16]. Given the presence of the same recombinant types in both the Chicago and Memphis cohorts, we propose that some of the mosaic viruses identified in this study might represent CRFs of FIV. However, we cannot exclude the possibility that the same recombinant forms could have arisen independently in distinct epidemiological areas on multiple occasions.

It is intriguing that clade A sequences were more likely to have a mosaic composition than those which were originally assigned to clade B. It has been proposed that clade B viruses are evolutionarily older, more host adapted and less pathogenic [20]. It is possible that the later emerging clade A viruses were able to superinfect cats and to recombine with already existing clade $\mathrm{B}$ viruses, resulting in the emergence of multiple Clade A/B recombinants in which the env gene predominantly comprised clade A derived sequences. These A/B recombinant strains hypothetically possessed equivalent or greater fitness compared to the parental A strains and were fixed in the population as CRFs of FIV. In contrast, A/B recombinants that were predominantly clade $B$ derived were conspicuously rare, suggesting that these recombinants incurred a fitness cost.

Our analyses revealed the existence of at least one common recombination break point, located at the stem of the V1/V2 loop of the SU of FIV Env. Common recombination breakpoints, or hotspots, have been reported in both FIV [16] and HIV [5,6,9,31-33] infections. The identification of common recombination hotspots was limited since only env sequences were examined and it is likely that recombination processes within env were accompanied by recombination events elsewhere in the genome, such that additional, as yet unidentified, breakpoints exist $[16,17]$.

Although recombination occurs frequently in vitro [34-36], it has been suggested that recombination breakpoints are driven by selection rather than being hotspots of RT template switching [4]. The vast majority of recombinant viruses display low fitness and do not survive within the host [37]. The recombinant env variants identified in our study must therefore have suffered no fitness cost, or might even have possessed some fitness advantage, compared to parental sequences.

The results of the present study highlight the risk of examining partial gene sequences. The detection of recombination increases with the nucleotide length screened and therefore the majority of previous studies (which examined only short 500-700 bp fragments of the env gene) underestimated the role of recombination using a simplified phylogenetic classification. Applying the common approach of examining the V3-V5 hypervariable regions of the env gene will likely result in the misidentification of intra- and inter-subtype recombinants and erroneous subtype assignment. For example, the recombinant structure of viruses such as M2, M33 or P7 would have been misclassified if only $700 \mathrm{bp}$ fragments of the env had been examined. Indeed, previous studies focusing solely on the V3-V5 region of env $[24,38]$ did not take into account the possibility of recombination which might have altered the classification of FIVs [17].

The recombination breakpoint separating the unusually long leader region from the remainder of the env gene is of particular interest. Several recombinant env sequences were identified in which the leader/signal region of env clustered with clade B while the other spans clustered with clade A. Given the location of this recombination breakpoint, together with the relatively high number of positively selected sites and the highest evolutionary rate of this span [28], the leader region may have a significant function in the viral life cycle and immune evasion.

Sequences isolated from the majority of animals formed highly monophyletic groups, suggesting that viruses were not transmitted between cats. Sequential sequences from three animals displayed incongruent phylogenetic assignment. Since the cats were housed together with other FIV infected cats, their shared accommodation may have led to infection with additional viruses, including transmission from unsampled cats, resulting in the potential turnover of the viral population. Given the relatively small number of sequences isolated following the postulated transmission event and sampling bias, it is possible that primary sequences remained but were less abundant than more recently acquired strains.

Potential limitations of this study include PCR sampling and cloning bias [39,40]. However, in contrast to 
previous studies, a high fidelity DNA polymerase (Phusion ${ }^{\odot}$ ) was used in place of the error prone Taq polymerase to avoid mutations arising during the PCR amplification of env sequences. Furthermore, in order to minimize template switching during the PCR, three independent amplifications were set up from each blood sample. The presence of similar recombinants, particularly those with shared recombination points, over a period of 18 months provides strong evidence that the amplicons identified were indeed circulating sequences rather than products of PCR-induced errors or polymerase template switching.

\section{Conclusions}

Recombinant env sequences sharing a common breakpoint separating the leader/signal region from the remaining part of env were highly abundant in naturally infected cats in the USA. This finding is intriguing, particularly since the feline and ungulate lentiviruses possess unusually long leader/signal sequences compared to primate retroviruses [18]. The location of the identified recombination breakpoint suggests that the leader region of the FIV env could play an important role in virus biology and immune evasion, as has been described for signal sequences in other viruses [41]. These findings broaden our understanding of retroviral evolution and illustrate the significant role of recombination in generating viral diversity at the population level. Here we provide evidence for the existence of CRFs in two geographically distant American cities. The lack of information about CRFs of FIV in the field has wider implications than just the classification of FIV, since it poses significant questions about the likely efficacy of the current FIV vaccine. The degree of protection against recombinant viruses provided by commercial FIV vaccination is unknown. Furthermore, the existence of recombinant sequences has implications for the molecular diagnosis of FIV infection. CRFs of FIV, including some as yet unidentified, may remain undetected by PCR-based diagnostic tests $[42,43]$ currently in use to distinguish FIV-vaccinated and FIV-infected cats in countries where the vaccine is widely available [44]. Further phylogenetic studies, ideally of two neighbouring genes or the whole genome of various strains of FIV from diverse geographic locations will be required to classify the virus more accurately, to optimize diagnostic protocols and to inform the development of an improved FIV vaccine.

\section{Methods}

\section{FIV ORF env sequences}

FIV were isolated from three serial blood samples collected at 6 monthly intervals from naturally infected domestic cats $(n=43)$. Cats were enrolled into the study based on a history of FIV diagnosis by ELISA (SNAP FIV/FeLV Combo Test, IDEXX), regardless of breed, age, sex or health status. All cats were FeLV antigen negative (SNAP FIV/FeLV Combo Test, IDEXX). FIVpositive status was confirmed by virus isolation [45]. Within the study group, 27 cats were homed together and cared for in a large multi-cat household in Memphis, TN, USA, where FIV-positive and FIV-negative cats were housed indoors with unrestricted access to one another. The remaining 16 cats were living in single households in Chicago, IL, USA with exception of five cats: two cats (P7 and P4) had been rehomed together and were living in the same household; one cat (P9) had been rehomed with another FIV-positive cat not enrolled in the study; and one cat (P13) had been rehomed with another FIV-negative cat; and one cat (P21) was housed with another two FIV-positive cats in the rehoming centre.

Cats from two cohorts displayed contrasting clinical outcomes of infection. The clinical signs were mild or unapparent in the Chicago cohort, while the outcome of infection in Memphis was dramatically different, with mortality rate reaching $63 \%$ and lymphoma being the most common cause of death [28].

The study and its aims were reviewed and approved by the University of Glasgow Ethics Committee and the Purdue Animal Care and Use Committee. Cat owners provided written informed consent for their participation in the study.

Multiple full length FIV env genes ( 2500 bp) were amplified directly from whole blood using a nested PCR protocol [see Additional file 7: Table S4]. First round PCR products were amplified by Phusion ${ }^{\oplus}$ Blood Direct II Polymerase (Thermo Fisher Scientific), followed by direct nucleic acid sequence determination. The nucleic acid sequence of the first-round PCR product informed primer design for the second round PCR, which was performed using High Fidelity PCR Master (Roche). Strain-specific primers for second round PCR incorporated restriction sites for subsequent cloning into the eukaryotic expression vector VR1012 [46] and transformation into E. coli MAX Efficiency ${ }^{\circ}$ D $5 \alpha^{\mathrm{TM}}$ Competent Cells (Invitrogen). Each VR1012-FIV env construct was sequenced using Big Dye Terminator v1.1 kit (Applied Biosystems) on an Applied Biosystems 3130xl capillary sequencer. Special measures were taken to avoid the possibility of contamination, both in the clinical and the laboratory settings: cats were double identified prior to blood sampling; PCR reactions were prepared in a designated UV treated room; and fresh, unopened reagents were used at each separate time point throughout the 18 month study period.

\section{Multiple sequence alignment}

There were 355 serial env sequences from 43 cats available for analysis from the two cohorts [see Additional file 5: Table S2]. The number of sequences varied according to 
the availability of follow-up samples, because of the $63 \%$ mortality rate in the Memphis cohort during the study period [28]. Multiple sequence alignments were conducted using the Muscle algorithm [47] in MEGA5 [48]. Final alignments were curated manually to ensure repositioning of incorrect gaps in sequences of variable lengths.

\section{Phylogenetic trees}

Maximum likelihood (ML) trees were constructed in MEGA5 [48] under the HKY nucleotide substitution model, selected through jMODELTEST [49]. Statistical support for the ML trees was estimated using 1000 bootstrap replicates [50].

\section{Recombination testing}

Sequences from the study group $(\mathrm{n}=355)$, together with reference full-length env sequences obtained from GenBank $(\mathrm{n}=19)$, were subjected to rigorous five-fold recombination testing: 1) The initial recombination analysis included the entire data set and was performed with the Datamonkey webserver [51], employing Single Breakpoint (SBP) and Genetic Algorithm Recombination Detection (GARD) methods [52] and using the Pairwise Homoplasy Index in SPLITSTREE [53]; 2) The mosaic structure of recombinant sequences was confirmed by subsequent GARD analysis focussing only on study group sequences identified previously as recombinants, to achieve higher resolution (maximum likelihood trees for each recombination span identified by GARD and assessed by Akaike Information Criterion (AIC) [54] were constructed on the Datamonkey webserver); 3) The mosaic structure of recombinant sequences was tested by employing probabilistic approach implemented in jpHMM [55]; 4) Major and minor parents for mosaic recombinant sequences were identified by the RDP detection method [56] and confirmed for the complete dataset by SPLITSTREE network [53]; and 5) Representative figures visualizing recombination breakpoints were generated in SimPlot v 3.5.1 [57].

\section{Additional files}

Additional file 1: The Maximum Likelihood (ML) tree constructed under HKY substitution model using the entire data set. The tree is saved in the NEWICK format and can be opened in FigTree $v$ 1.3.1 (http://tree.bio.ed.ac.uk/).

Additional file 2: Figure S1. ML trees representing phylogenetic inference of seven GARD determined spans.

Additional file 3: Table S1. Comparison of GARD and jpHMM identified recombination breakpoints.

Additional file 4: Figure S2. Similarity analysis of: A) Memphis A/B recombinant sequence (M31) and B) Chicago $A / B$ recombinant sequence (P21C).

Additional file 5: Table S2. Number of sequences isolated from each time point from the US cats.
Additional file 6: Table S3. FIV load, (genomes/mL blood).

Additional file 7: Table S4. Primers.

\section{Abbreviations}

FIV: Feline immunodeficiency virus; RT: Reverse transcriptase; URF: Unique recombinant form; CRF: Commonly circulating recombinant form; GARD: Genetic algorithm recombination detection; AIC: Akaike information criterion; SBP: Single breakpoint; ML: Maximum likelihood; bp: Base pair.

\section{Competing interests}

The authors declare that they have no competing interests.

\section{Authors' contributions}

PB carried out amplifications, cloning, sequencing, alignments, data analyses, coordinated the study and drafted the manuscript. JH and RB contributed to the design and implementation of the data analysis and drafted the manuscript. AL carried out clinical examinations, collected the blood samples, coordinated logistics and drafted the manuscript. MJH and BJW conceived the study, participated in its design and coordination and drafted the manuscript. All authors read and approved the final manuscript.

\section{Acknowledgements}

This study was supported by The Wellcome Trust. Blood sample collections were supported by the Purdue Maddie's Shelter Medicine Program underwritten by a grant from Maddie's Fund, The Pet Rescue Foundation (www.maddiesfund.org), helping to fund the creation of a no-kill nation. RB is supported by the RAPIDD programme of the Science and Technology Directorate of the Department of Homeland Security and NIH Fogarty International Center. We thank Dr James Mullins and Breana Hall from Centre for AIDS Reasearch (CFAR) of the University of Washington for performing jpHMM analyses. CFAR is supported by NIAID, NCI, NIMH, NIDA, NICHD, NHLBI, NIA, NIGMS, NIDDK of the National Institutes of Health under award number P30AI027757. We thank Kristen Hall CVT, Dr Jui Ming Lin, Dr Christian Leutenegger, PAWS Chicago, Drennan Animal Hospital, the Fitzhugh B. Crews FIV Cat Sanctuary and participating cat owners for their assistance with the study. We thank IDEXX Corporation (Westbrook, MN and West Sacramento, CA) for providing the FIV load and flow cytometry results.

\section{Author details}

${ }^{1}$ Centre for Virus Research, University of Glasgow, Glasgow, United Kingdom. ${ }^{2}$ Small Animal Hospital, University of Glasgow, Glasgow, United Kingdom. ${ }^{3}$ Boyd Orr Centre for Population and Ecosystem Health \& Institute of Biodiversity, Animal Health \& Comparative Medicine, University of Glasgow, Glasgow, United Kingdom. ${ }^{4}$ Department of Veterinary Clinical Sciences, Purdue University, West Lafayette, IN 47907, USA.

Received: 23 April 2014 Accepted: 1 September 2014 Published online: 17 September 2014

\section{References}

1. Preston BD, Dougherty JP: Mechanisms of retroviral mutation. Trends Microbiol 1996, 4:16-21.

2. Chiu Y-L, Greene WC: Multifaceted antiviral actions of APOBEC3 cytidine deaminases. Trends Immunol 2006, 27:291-297.

3. Charpentier C, Nora T, Tenaillon O, Clavel F, Hance AJ: Extensive recombination among human immunodeficiency virus type 1 quasispecies makes an important contribution to viral diversity in individual patients. J Virol 2006, 80:2472-2482.

4. Onafuwa-Nuga A, Telesnitsky A: The remarkable frequency of human immunodeficiency virus type 1 genetic recombination. Microbiol Mol Biol Rev 2009, 73:451-480.

5. Zhuang J, Jetzt AE, Sun G, Yu H, Klarmann G, Ron Y, Preston BD, Dougherty JP: Human immunodeficiency virus type 1 recombination: rate, fidelity, and putative hot spots. J Virol 2002, 76:11273-11282.

6. Levy DN, Aldrovandi GM, Kutsch O, Shaw GM: Dynamics of HIV-1 recombination in its natural target cells. Proc Natl Acad Sci 2004 101:4204-4209.

7. Basu VP, Song M, Gao L, Rigby ST, Hanson MN, Bambara RA: Strand transfer events during HIV-1 reverse transcription. Virus Res 2008, 134:19-38. 
8. Kuwata T, Miyazaki Y, Igarashi T, Takehisa J, Hayami M: The rapid spread of recombinants during a natural in vitro infection with two human immunodeficiency virus type 1 strains. J Virol 1997, 71:7088-7091.

9. Jetzt $A E, Y u H$, Klarmann GJ, Ron Y, Preston BD, Dougherty JP: High rate of recombination throughout the human immunodeficiency virus type 1 genome. J Virol 2000, 74:1234-1240.

10. Blackard JT, Cohen DE, Mayer KH: Human immunodeficiency virus superinfection and recombination: current state of knowledge and potential clinical consequences. Clin Infect Dis 2002, 34:1108-1114.

11. Tebit DM, Nankya I, Arts EJ, Gao Y: HIV diversity, recombination and disease progression: how does fitness "fit" into the puzzle? AIDS Rev 2007, 9:75-87.

12. Robertson DL, Anderson JP, Bradac JA, Carr JK, Foley B, Funkhouser RK, Gao F, Hahn BH, Kalish ML, Kuiken C, Learn GH, Leitner T, McCutchan F, Osmanov S, Peeters M, Pieniazek D, Salminen M, Sharp PM, Wolinsky S, Korber B: HIV-1 nomenclature proposal. Science 2000, 288:55-56.

13. Peeters M, Sharp PM: Genetic diversity of HIV-1: the moving target. AIDS 2000, 14(Suppl 3):S129-S140.

14. Bachmann MH, Mathiason-Dubard C, Learn GH, Rodrigo AG, Sodora DL, Mazzetti P, Hoover EA, Mullins Jl: Genetic diversity of feline immunodeficiency virus: dual infection, recombination, and distinct evolutionary rates among envelope sequence clades. J Virol 1997, 71:4241-4253.

15. Carpenter MA, Brown EW, MacDonald DW, O'Brien SJ: Phylogeographic patterns of feline immunodeficiency virus genetic diversity in the domestic cat. Virology 1998, 251:234-243.

16. Reggeti F, Bienzle D: Feline immunodeficiency virus subtypes A, B and C and intersubtype recombinants in Ontario, Canada. J Gen Virol 2004 85:1843-1852

17. Hayward JJ, Rodrigo AG: Recombination in feline immunodeficiency virus from feral and companion domestic cats. Virol J 2008, 5:76.

18. Verschoor EJ, Hulskotte EG, Ederveen J, Koolen MJ, Horzinek MC, Rottier PJ: Post-translational processing of the feline immunodeficiency virus envelope precursor protein. Virology 1993, 193:433-438.

19. Nishimura $Y$, Goto $Y$, Pang $H$, Endo $Y$, Mizuno T, Momoi $Y$, Watari T, Tsujimoto $H$, Hasegawa A: Genetic heterogeneity of env gene of feline immunodeficiency virus obtained from multiple districts in Japan. Virus Res 1998, 57:101-112.

20. Sodora DL, Shpaer EG, Kitchell BE, Dow SW, Hoover EA, Mullins Jl: Identification of three feline immunodeficiency virus (FIV) env gene subtypes and comparison of the FIV and human immunodeficiency virus type 1 evolutionary patterns. J Virol 1994, 68:2230-2238.

21. Kann RKC, Kyaw-Tanner MT, Seddon JM, Lehrbach PR, Zwijnenberg RJG, Meers J: Molecular subtyping of feline immunodeficiency virus from domestic cats in Australia. Aust Vet J 2006, 84:112-116.

22. Samman A, McMonagle EL, Logan N, Willett BJ, Biek R, Hosie MJ: Phylogenetic characterisation of naturally occurring feline immunodeficiency virus in the United Kingdom. Vet Microbio/ 2011, 150:239-247.

23. Steinrigl A, Klein D: Phylogenetic analysis of feline immunodeficiency virus in Central Europe: a prerequisite for vaccination and molecular diagnostics. J Gen Virol 2003, 84:1301-1307.

24. Duarte A, Tavares L: Phylogenetic analysis of Portuguese feline immunodeficiency virus sequences reveals high genetic diversity. Vet Microbiol 2006, 114:25-33.

25. Uema M, Ikeda Y, Miyazawa T, Lin JA, Chen MC, Kuo TF, Kai C, Mikami T, Takahashi E: Feline immunodeficiency virus subtype $C$ is prevalent in northern part of Taiwan. J Vet Med Sci 1999, 61:197-199.

26. Hohdatsu T, Hirabayashi H, Motokawa K, Koyama H: Comparative study of the cell tropism of feline immunodeficiency virus isolates of subtypes $A$, $B$ and $D$ classified on the basis of the env gene V3-V5 sequence. $J$ Gen Virol 1996, 77(Pt 1):93-100.

27. Pecoraro MR, Tomonaga K, Miyazawa T, Kawaguchi Y, Sugita S, Tohya Y, Kai C, Etcheverrigaray ME, Mikami T: Genetic diversity of Argentine isolates of feline immunodeficiency virus. J Gen Virol 1996, 77(Pt 9):2031-2035.

28. Bęczkowski PM: Virus evolution in the progression of natural feline immunodeficiency virus infection. PhD Thesis. University of Glasgow, Centre for Virus Research; 2013.

29. Okada S, Pu R, Young E, Stoffs WV, Yamamoto JK: Superinfection of cats with feline immunodeficiency virus subtypes A and B. AIDS Res Hum Retroviruses 1994, 10:1739-1746.

30. Kyaw-Tanner MT, Greene WK, Park HS, Robinson WF: The induction of in vivo superinfection and recombination using feline immunodeficiency virus as the model. Arch Virol 1994, 138:261-271.
31. Anderson JA, Bowman EH, Hu W-S: Retroviral recombination rates do not increase linearly with marker distance and are limited by the size of the recombining subpopulation. J Virol 1998, 72:1195-1202.

32. Wooley DP, Bircher LA, Smith RA: Retroviral recombination is nonrandom and sequence dependent. Virology 1998, 243:229-234

33. Dykes C, Balakrishnan M, Planelles V, Zhu Y, Bambara RA, Demeter LM: Identification of a preferred region for recombination and mutation in HIV-1 gag. Virology 2004, 326:262-279.

34. An W, Telesnitsky A: HIV-1 genetic recombination: experimental approaches and observations. AIDS Rev 2002, 4:195-212.

35. Rhodes T, Wargo H, Hu W-S: High rates of human immunodeficiency virus type 1 recombination: near-random segregation of markers one kilobase apart in one round of viral replication. J Virol 2003, 77:11193-11200.

36. Chen J, Powell D, Hu W-S: High frequency of genetic recombination is a common feature of primate lentivirus replication. J Virol 2006 80:9651-9658

37. Archer J, Pinney JW, Fan J, Simon-Loriere E, Arts EJ, Negroni M, Robertson DL: Identifying the important HIV-1 recombination breakpoints. PLOS Comput Biol 2008, 4:e1000178.

38. Weaver EA, Collisson EW, Slater M, Zhu G: Phylogenetic analyses of Texas isolates indicate an evolving subtype of the clade B feline immunodeficiency viruses. J Virol 2004, 78:2158-2163.

39. Simmonds $P$, Balfe $P$, Peutherer JF, Ludlam CA, Bishop JO, Brown AJ: Human immunodeficiency virus-infected individuals contain provirus in small numbers of peripheral mononuclear cells and at low copy numbers. J Virol 1990, 64:864-872.

40. Liu SL, Rodrigo AG, Shankarappa R, Learn GH, Hsu L, Davidov O, Zhao LP, Mullins Jl: HIV quasispecies and resampling. Science 1996, 273:415-416.

41. Hegde RS, Bernstein HD: The surprising complexity of signal sequences. Trends Biochem Sci 2006, 31:563-571.

42. Bienzle D, Reggeti F, Wen X, Little S, Hobson J, Kruth S: The variability of serological and molecular diagnosis of feline immunodeficiency virus infection. Can Vet J 2004, 45:753-757

43. Crawford PC, Slater MR, Levy JK: Accuracy of polymerase chain reaction assays for diagnosis of feline immunodeficiency virus infection in cats. J Am Vet Med Assoc 2005, 226:1503-1507.

44. Little S, Bienzle D, Carioto L, Chisholm H, O'Brien E, Scherk M: Feline leukemia virus and feline immunodeficiency virus in Canada: recommendations for testing and management. Can Vet J 2011, 52:849-855.

45. Hosie MJ, Addie D, Belák S, Boucraut-Baralon C, Egberink H, Frymus T, Gruffydd-Jones T, Hartmann K, Lloret A, Lutz H, Marsilio F, Pennisi MG, Radford AD, Thiry E, Truyen U, Horzinek MC: Feline immunodeficiency. ABCD guidelines on prevention and management. J Feline Med Surg 2009, 11:575-584.

46. Hartikka J, Sawdey M, CornefertJensen F, Margalith M, Barnhart K, Nolasco M, Vahlsing HL, Meek J, Marquet M, Hobart P, Norman J, Manthorpe M: An improved plasmid DNA expression vector for direct injection into skeletal muscle. Hum Gene Ther 1996, 7:1205-1217.

47. Edgar RC: MUSCLE: multiple sequence alignment with high accuracy and high throughput. Nucleic Acids Res 2004, 32:1792-1797.

48. Tamura K, Peterson D, Peterson N, Stecher G, Nei M, Kumar S: MEGA5: molecular evolutionary genetics analysis using maximum likelihood, evolutionary distance, and maximum parsimony methods. Mol Biol Evol 2011, 28:2731-2739

49. Posada D: jModelTest: phylogenetic model averaging. Mol Biol Evol 2008 , 25:1253-1256.

50. Efron B, Halloran E, Holmes S: Bootstrap confidence levels for phylogenetic trees. Proc Natl Acad Sci U S A 1996, 93:13429-13434.

51. Delport W, Poon AF, Frost SD, Kosakovsky Pond SL: Datamonkey 2010: a suite of phylogenetic analysis tools for evolutionary biology. Bioinformatics 2010, 26:2455-2457.

52. Kosakovsky Pond SL, Posada D, Gravenor MB, Woelk CH, Frost SD: Automated phylogenetic detection of recombination using a genetic algorithm. Mol Biol Evol 2006, 23:1891-1901.

53. Huson DH, Bryant D: Application of phylogenetic networks in evolutionary studies. Mol Biol Evol 2006, 23:254-267.

54. Sugiura N: Further analysts of the data by akaike's information criterion and the finite corrections. Commun Stat - Theory Methods 1978, 7:13-26.

55. Schultz AK, Zhang M, Leitner T, Kuiken C, Korber B, Morgenstern B, Stanke M: A jumping profile hidden Markov model and applications to 
recombination sites in HIV and HCV genomes. BMC Bioinformatics 2006, 7:265.

56. Martin DP, Lemey P, Lott M, Moulton V, Posada D, Lefeuvre P: RDP3: a flexible and fast computer program for analyzing recombination. Bioinformatics 2010, 26:2462-2463.

57. Lole KS, Bollinger RC, Paranjape RS, Gadkari D, Kulkarni SS, Novak NG, Ingersoll R, Sheppard HW, Ray SC: Full-length human immunodeficiency virus type 1 genomes from subtype C-infected seroconverters in India, with evidence of intersubtype recombination. J Virol 1999, 73:152-160.

doi:10.1186/s12977-014-0080-1

Cite this article as: Bęczkowski et al:: Feline immunodeficiency virus (FIV) env recombinants are common in natural infections. Retrovirology 2014 11:80.

\section{Submit your next manuscript to BioMed Central and take full advantage of:}

- Convenient online submission

- Thorough peer review

- No space constraints or color figure charges

- Immediate publication on acceptance

- Inclusion in PubMed, CAS, Scopus and Google Scholar

- Research which is freely available for redistribution 